Copyright (C 2006 IEEE. Reprinted from IEEE Photonics Technology Letters, 2004; 16 (11):2502-2504

This material is posted here with permission of the IEEE. Such permission of the IEEE does not in any way imply IEEE endorsement of any of the University of Adelaide's products or services. Internal or personal use of this material is permitted. However, permission to reprint/republish this material for advertising or promotional purposes or for creating new collective works for resale or redistribution must be obtained from the IEEE by writing to pubs-permissions@ieee.org.

By choosing to view this document, you agree to all provisions of the copyright laws protecting it. 


\title{
High Absorption and Low Splice Loss Properties of Hexagonal Double-Clad Fiber
}

\author{
Yahua Li, Student Member, IEEE, Stuart D. Jackson, and Simon Fleming
}

\begin{abstract}
An accurate experimental comparison of the pump absorption and the lasing features between $\mathrm{Nd}^{3+}$-doped fiber lasers using hexagonal-shaped and D-shaped fiber is reported. The measured slope efficiencies with respect to the absorbed pump power of $805 \mathrm{~nm}$ are nearly identical at $\sim 65.3 \%$ for both fibers. Taking advantage of the near symmetrical shape of the hexagonal fiber, the lowest reported splice loss of $0.05 \mathrm{~dB}$ is achieved between the double-clad fiber and standard $125-\mu \mathrm{m}$ fiber with the use of the fiber etching techniques. The pump absorption can be improved by bending the hexagonal fibers into a kidney shape.
\end{abstract}

Index Terms-Absorption, cross section, double-clad fiber (DCF), hexagonal, splice.

\section{INTRODUCTION}

B ECAUSE OF the high efficiency, high power, and favorable thermal management, rare-earth-doped fiber lasers are one of the most attractive laser devices for applications in sensors, medicine, optical communications, and industrial processing. To overcome the difficulty of coupling the pump power into the active core, double-clad fibers (DCFs) are designed to support good conversion from diode laser light to single-mode radiation and allow easy scaling of the output power [1], [2]. The optical efficiency of a fiber laser, therefore, critically depends on the coupling of the injected pump light from the pump core to the active core. To date, there are a variety of cladding geometries to improve pump absorption, e.g., circular offset, rectangular, D-shaped, hexagonal, flower, and a circular cladding with an inner D-shaped hole [3]-[8]. D-shaped fiber (DSF) has been widely used in high-power double-clad fiber lasers (DCFL) because of its high pump absorption efficiency and low production cost as compared with rectangular and circular offset core cross-sectional fibers [8]. Furthermore, as simulated in [6], the flower-shaped cross-sectional fiber can provide similar pump absorption features to the DSF. In comparison with DSF, the hexagonal-shaped fiber (HSF) has the additional advantage of a more symmetrical cross section, which is critical when low loss splicing between two different fiber geometries is required. To our knowledge, however, there

Manuscript received June 2, 2004; revised June 30, 2004. This work was supported by the Australian Research Council and by the Australian Photonics Co-operative Research Centre. The work of Y. H. Li was supported by the International Postgraduate Research Scholarship (IPRS), funded by the Commonwealth Department of Education, Science and Training (DEST).

Y. Li is with the Optical Fiber Technology Centre and the School of Electrical and Information Engineering, University of Sydney, Sydney 2006, Australia (e-mail: y.li@oftc.usyd.edu.au).

S. D. Jackson and S. Fleming are with the Optical Fiber Technology Centre, Australian Photonics CRC, University of Sydney, 1430 Eveleigh, Sydney, Australia.

Digital Object Identifier 10.1109/LPT.2004.834919

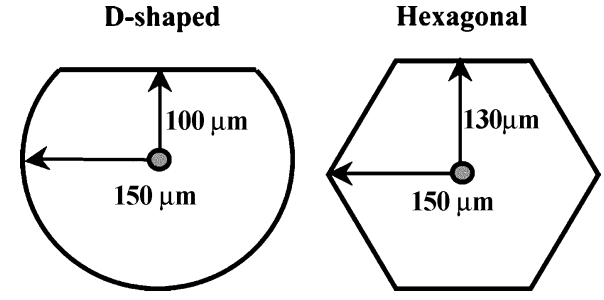

Fig. 1. Cross-sectional views of DSF and HSF.

has been no report on the comparison between DSF and HSF. In this letter, we first experimentally compared the absorption characteristics of HSF and DSF fibers. In order to obtain an accurate comparison, we made the two fibers from the same preform to provide the same core parameters. The cross sections of both fibers are shown schematically in Fig. 1. Due to the large number of skew rays in a circular DCF with centered core, only a part of the pump light is absorbed in the active core. Normally, tightly bending a circular fiber can improve the scrambling of the pump light and hence improve the pump absorption [8], [9]. For this reason, fiber with a broken circular geometry is considered to provide more pump absorption, with a simultaneous increase of the laser output power. However, we found in the experiment that bending HSF could also improve the absorption of the pump light and, hence, increase the output power at the same launched pump level. Finally, a remarkably low loss splice of $0.05 \mathrm{~dB}$ between a broken circular geometry double-clad HSF and standard $125-\mu \mathrm{m}$ fiber was obtained with the use of the fiber fusion splicing and etching techniques.

\section{EXPERIMENTS AND RESULTS}

In our experiment, the $\mathrm{Nd}^{3+}$-doped preform was fabricated from the standard modified chemical vapor deposition and the solution doping techniques. Before being drawn into fiber, the preform was divided into two sections and milled to the required cross sections, as shown in Fig. 1. The dimensions of both HSF and DSF are also shown in Fig. 1. The area of DSF pump core was 7\% larger than the corresponding area of HSF. The fibers had an active core diameter of $3.48 \mu \mathrm{m}$ with numerical aperture (NA) of 0.16 , providing a $\sim 730$-nm cutoff wavelength. The pump core (both fibers had a maximum dimension of $300 \mu \mathrm{m}$ ) was surrounded by a low index ultraviolet-curable fluoro-polymer to create an NA of 0.35 for the pump light. The measured concentration of $\mathrm{Nd}^{3+}$ ions was $\sim 2200 \mathrm{ppm}$. The fibers used in the experiment have a length of $21.5 \mathrm{~m}$. Fig. 2 shows schematically the experimental configuration. The fiber laser was forward pumped with a high-power continuous-wave 


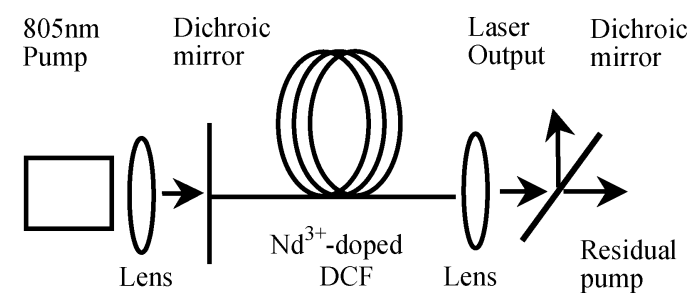

Fig. 2. Schematic diagram of the $\mathrm{Nd}^{3+}$-doped DCF laser configuration.

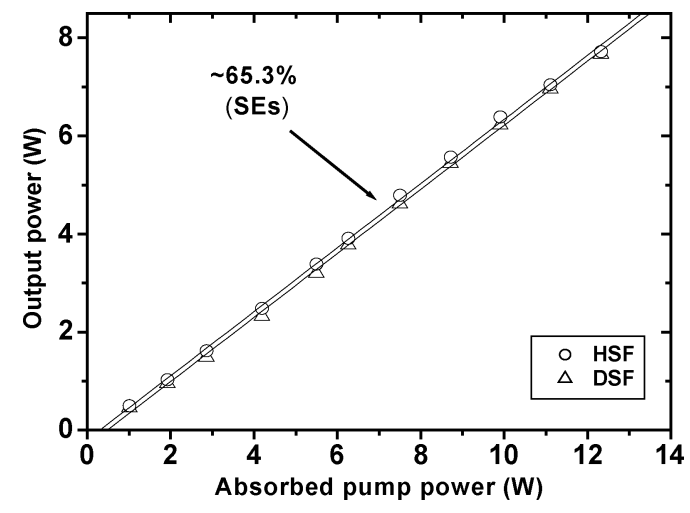

Fig. 3. Output power as a function of absorbed pump power at $805 \mathrm{~nm}$.

diode laser system operating at a wavelength of $805 \mathrm{~nm}$. An $f=30 \mathrm{~mm}$ multilens focus system was used to efficiently launch the pump light into the pump core and the measured launch efficiency for both of HSF and DSF was 70\%. The incident pump power intensity is $\sim 60 \mathrm{~kW} / \mathrm{cm}^{2}$ onto the pump core. A broad-band dielectric mirror butted against the input end to the fiber and Fresnel reflection at the output end to the fiber formed the laser resonator. The output power was measured with an Ophir Orion or a Melles Griot 13PEM001 power meter after the output from the fiber laser was collimated and the laser and the residual pump were separated with the use of a lens and a $45^{\circ}$ dichroic mirror $(R>99 \%$ at $1.06 \mu \mathrm{m}$ and $R<5 \%$ at $805 \mathrm{~nm}$ ). The output optical spectrum was measured with an Anritsu MS9710C spectrum analyzer.

Fig. 3 shows the output powers measured as a function of absorbed pump power for fiber lasers using either HSF or DSF. The absorbed pump power was determined by measuring the residual pump as a function of the launched pump power. In order to minimize the bending loss, both fibers were coiled with radii of $\sim 300 \mathrm{~mm}$. The measured effective absorption coefficients for HSF and DSF were 0.041 and $0.040 \mathrm{~m}^{-1}$, respectively. The measured slope efficiencies (SEs) for both HSF and DSF corresponding to the absorbed pump power were nearly identical at $\sim 65.3 \%$, and the laser thresholds measured were also similar ( $\sim 0.12-\mathrm{W}$ absorbed pump power). This can be explained from the fact that the total pump losses along the fiber are the same for both fibers. The total pump losses were attributed to 1) the pump absorption in the active core and 2) the pump losses as a result of scattering and leakage at the pump core-polymer boundaries. Since both fibers were made from the same preform, the pump absorption in the active core is assumed to be the same for the two fibers. The losses at the pump core-polymer boundaries can be considered to be the same for both fiber geometries.

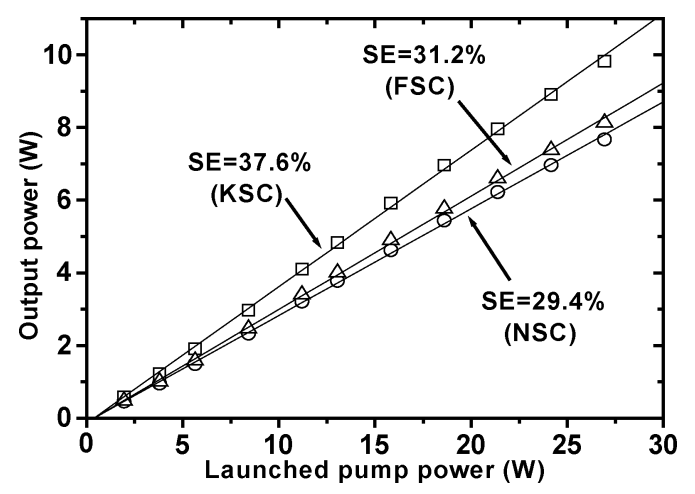

Fig. 4. Output power as a function of launched pump power for different coilings.

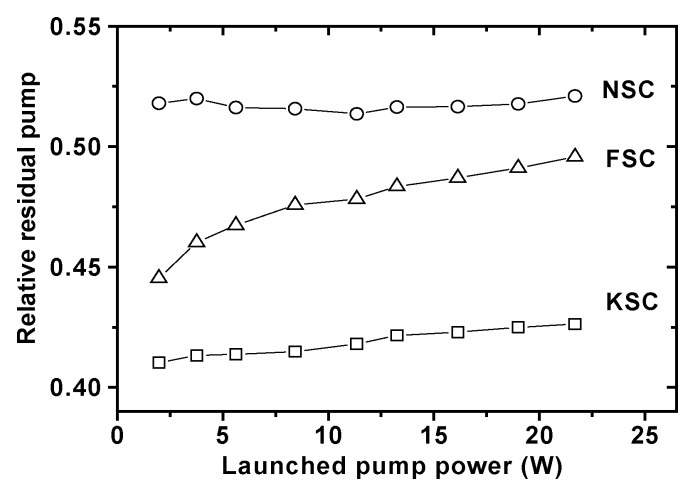

Fig. 5. Relative residual pump with respect to launched pump power for different coilings.

We also improved the pump absorption by bending HSF into different shapes. The pump absorption in HSF was strongly dependent on the coil shape of the fiber. We coiled 21.5-m HSF into three configurations: kidney shape coiling (KSC), figureeight shape coiling (FSC), and normal spool coiling (NSC). In cases of the KSC and FSC, the bending radii were $20 \mathrm{~mm}$. Fig. 4 shows the measured SEs of the KSC, FSC, and NSC as functions of launched pump power measured from the three fibers coiled into different shapes. As we expected, the KSC has the highest SE, since more pump power was absorbed with KSC. The 21.5-m length used in the experiments was significantly shorter than the optimal length and, thus, these SEs were much lower, as compared with those relating to the absorbed pump power.

Fig. 5 shows the dependences of the relative residual pump $P_{r} / P_{l}$ (where $P_{r}$ and $P_{l}$ are the residual pump and the launched pump power, respectively) on the launched pump power for the same set of parameters as used in Fig. 4. The KSC provided the best pump scrambling and the highest pump absorption amongst the three coils tested, while there is only slight improvement of absorption in the FSC, compared with the NSC. In KSC, the pump trajectory was periodically varied at the point where the curvature changes, while there was less curvature variation associated with FSC. The more change in the pump trajectory, the more absorption occurred in the active core. Unlike the absorption properties associated with KSC and NSC, the absorption in FSC significantly decreased at higher pump powers. This phenomenon is currently under investigation. We could not compare the absorption features between DSF and HSF under var- 
ious bending regimes because of the fragility associated with the nonsymmetrical cross section of DSF.

For a rare-earth-doped DCFL incorporating FBG resonators, the DCF is typically spliced to a short piece of standard $125-\mu \mathrm{m}$ fiber that has an FBG written in the core. Since the splice occurs within the resonator, a good splice with a very low loss is critical. Unfortunately, standard techniques for cleaving and low-loss splicing are not always effective with these nonstandard fibers and special techniques such as fattened or tapered fusion fibers and thermally expanded core fibers [10], [11] have to be developed to achieve as low as possible splice loss. Taking advantage of the symmetrical geometrical cross section of HSF, however, low splicing loss can be obtained by etching the bigger HSF fiber to closely match the diameter of $125-\mu \mathrm{m}$ fiber. Briefly, after a $10-\mathrm{cm}$ section of the output end of HSF has been etched for $47 \mathrm{~min}$ with the use of HF acid, the original dimensions of $300 / 260-\mu \mathrm{m}$ (pump core) for the longer/shorter axis decreased to $\sim 140 / 100 \mu \mathrm{m}$ to closely match the diameter of $125 \mu \mathrm{m}$ relevant to mode-matched photosensitive fiber. Due to the highly nonsymmetrical cross section of DSF, the minimum etchable dimensions were $\sim 165 / 115 \mu \mathrm{m}$. With the use of the techniques, a minimum 0.05-dB fusion splicing loss between HSF and standard $125 \mu \mathrm{m}$ fiber could be obtained repeatedly, while a minimum for DSF was $0.15 \mathrm{~dB}$. Misalignment of the DSF occurred frequently during the fusion splicing process. The splice loss was determined by measuring the output power at $1060 \mathrm{~nm}$ before and after splicing.

\section{CONCLUSION}

We have demonstrated experimentally that the absorption properties of HSF and DSF drawn from the same preform are nearly identical. Thus, the pump losses at the pump corepolymer boundaries are the same for both fiber geometries. While DSF provides a more chaotic pump trajectory, HSF provides similar absorption performance, and has the added advantage of being more symmetrical, which is an important feature for achieving low splice loss when incorporating an FBG into the resonator. With the use of the fiber etching technique, a minimum $0.05-\mathrm{dB}$ fusion splice loss between the hexagonal DCF and standard $125-\mu \mathrm{m}$ fiber was achieved in the experiments, while a minimum for DSF was $0.15 \mathrm{~dB}$. Bending the hexagonal fiber into a kidney-shape can improve the pump absorption, with a simultaneous increase in laser output power.

\section{ACKNOWLEDGMENT}

The authors would like to thank Dr. J. Nilsson for his useful discussions and also A. Michie for teaching his splicing techniques.

\section{REFERENCES}

[1] J. Limpert, A. Liem, H. Zellmer, and A. Tunnermann, "500 W continuous-wave fiber laser with excellent beam quality," Electron. Lett., vol. 39, no. 8, pp. 645-647, 2003.

[2] Y. Jeong, J. K. Sahu, R. B. Williams, D. J. Richardson, K. Furusawa, and J. Nilsson, "Ytterbium-doped large-core fiber laser with $272 \mathrm{~W}$ output power," Electron. Lett., vol. 39, no. 13, pp. 977-978, 2003.

[3] A. Liu, J. Song, K. Kamatani, and K. Ueda, "Rectangular double-clad fiber laser with two end-bundled pump," Electron. Lett., vol. 32, no. 18, pp. 1673-1674, 1996

[4] S. D. Jackson and Y. H. Li, "Multi-wavelength diode-cladding pumped $\mathrm{Nd}^{3+}$-doped germano-aluminosilicate fiber laser," IEEE J. Quantum Electron., vol. 39, pp. 1118-1122, Sept. 2003.

[5] G. Xia, J. Duan, S. Zhao, and S. Dong, "Light propagation in the multimode double-clad erbium: ytterbium co-doped fiber: theory and experiment," Opt. Laser Technol., vol. 36, no. 4, pp. 273-277, 2004.

[6] P. Leproux, S. Fevrier, V. Doya, P. Roy, and D. Pagnoux, "Modelingand optimization of double-clad fiber amplifiers using chaotic propagation of the pump," Opt. Fiber Technol., vol. 6, pp. 324-329, 2001.

[7] A. Martinez-Rios, A. N. Starodumov, H. Po, Y. Wang, A. A. Demidov, and $\mathrm{X}$. Li, "Efficient operation of double-clad $\mathrm{Yb}^{3+}$-doped fiber lasers with a novel circular cladding geometry," Opt. Lett., vol. 28, no. 18, pp. 1642-1644, 2003.

[8] H. Zellmer, A. Tünnermann, H. Welling, and V. Reichel, "Double-clad fiber laser with $30 \mathrm{~W}$ output power," in Optical Amplifiers and Their Applications, Optical Society America, Trends Optics and Photonics (OSA TOPs), vol. 16, 1997, pp. 137-140.

[9] J. Nilsson, S. Alam, J. A. Alvarez-Chavez, P. W. Turner, W. A. Clarkson, and A. B. Grudinin, "High-power and tunable operation of erbium-ytterbium co-doped cladding-pumped fiber lasers," IEEE J. Quantum Electron., vol. 39, pp. 987-994, Aug. 2003.

[10] E. M. O'Brien and C. D. Hussey, "Low-loss fattened fusion splices between different fibers," Electron. Lett., vol. 35, no. 2, pp. 168-169, 1999.

[11] Y. Ohtera, O. Hanaizumi, and S. Kawakami, "Numerical analysis of eigenmodes and splice losses of thermally diffused expanded core fibers," J. Lightwave Technol., vol. 17, pp. 2675-2682, Dec. 1999. 